prior EMR in both groups. CR-D in Males was $84 \%$ and CR-IM $80 \%$. In females CR-D was $86 \%$ and CR-IM $64 \%$ and not significantly different ( $\mathrm{p}=0.61$ and $\mathrm{p}=0.22$, respectively). Progression to cancer was 3\% in both cohorts at 12 months. There were 21 patients from both groups with recurrent dysplasia on follow up biopsy after successful treatment. Median time to recurrence in these after successful RFA was 380 days (IQR 177-615). Twenty recurrences were in males compared to one in female group which was statistically significant $(\mathrm{p}=0.04)$. There were 11 recurrences of IM alone in patients who had confirmed CR-IM at 12 months. All were in male patients (median time to recurrence of 626 days, IQR 237-822). Baseline BE length, histology, prior EMR did not influence risk of recurrence of dysplasia or IM.

Conclusion RFA for BE related neoplasia is equally effective in both males and females. Recurrence of neoplasia after successful eradication although uncommon overall is more common in males. The much lower recurrence rate in women raises the possibility that they could be discharged from follow up after successful treatment or have prolonged surveillance intervals compared to men. This could reduce the burden of surveillance endoscopy on overstretched services. All collaborators of UK RFA registry are acknowledged for their contributions to this work.

Disclosure of Interest None Declared.

\section{PTU-172 TREATMENT OUTCOMES FOR BARRETT'S OESOPHAGUS RELATED NEOPLASIA HAVE IMPROVED OVER TIME WITH CHANGES IN ENDOSCOPIC PRACTICE: FIVE YEAR EXPERIENCE FROM THE FIRST FIVE HUNDRED PATIENTS IN THE UNITED KINGDOM REGISTRY}

${ }^{1,2}$ RJ Haidry** ${ }^{1} \mathrm{M}$ Banks, ${ }^{1} \mathrm{~A}$ Gupta, ${ }^{2} \mathrm{M}$ Butt ${ }^{3} \mathrm{G}$ Fullarton, ${ }^{4} \mathrm{H}$ Smart ${ }^{3} \mathrm{H}$ Morris, ${ }^{5} \mathrm{R}$ Willert ${ }^{6} \mathrm{R}$ Narayanasamy, ${ }^{1} \mathrm{M}$ Rodriguez-Justo, ${ }^{1} \mathrm{M}$ Novelli, $1,2^{2} \mathrm{~L}$ Lovat. ${ }^{1} \mathrm{UCLH}$, London, UK: ${ }^{2} N M L C$, UCL, London, UK; ${ }^{3} G R I$, Glasgow, UK; ${ }^{4} R L U H$, Liverpool, UK; ${ }^{5} M R I$, Manchester, UK; ${ }^{6}$ St James Hospital, Dublin, Ireland

10.1136/gutjnl-2014-307263.246

Introduction Barrett's oesophagus (BE) is the recognised pre-cursor to oesophageal adenocarcinoma (OAC). Combined endotherapy with endoscopic mucosal resection (EMR) and Radiofrequency ablation (RFA) have emerged as alternatives to surgery for curative treatment of patients with BE related neoplasia over the past 5 years.

Methods We examine prospective data from United Kingdom (UK) registry of patients undergoing RFA/EMR for early neoplasia arising in BE since the launch of the registry in 2008. Primary outcomes for clearance of dysplasia (CR-D) and BE (CR-IM) at 12 months were assessed over two time periods, between 2008-2010 and from 2011-2013. In addition durability of successful treatment, progression to invasive OAC and changes in endoscopic practices were also analysed between the time periods. Before RFA, visible lesions were removed by EMR. Thereafter patients underwent RFA every 3 monthly and biopsies were taken at 12 months. New lesions appearing during RFA treatment were removed by rescue EMR. This treatment algorithm has remained unchanged over past 5 years.

Results We report on 510 patients who have completed treatment with 12 month histology over past 5 years. CR-D and CR-IM have improved significantly between the former and later time periods from $77 \%$ and $56 \%$ to $91 \%$ and $82 \%$ respectively ( $<<0.0001$ ). The use of EMR for visible lesions prior to initiating RFA has also increased from $48 \%$ to $60 \%(\mathrm{p}=0.013)$. Conversely need for rescue EMR has decreased significantly to $3 \%$ over the last two and half years compared to $13 \%$ during initial time period ( $\mathrm{p}<$ 0.0001 ). Progression to invasive OAC is not significantly different (2.8\% in $2011-2013$ vs. $4 \% 2008-2010, \mathrm{p}=0.56)$.

Conclusion We report one of the largest series of patients undergoing RFA for BE neoplasia. Clinical outcomes have improved significantly over the past 5 years as endoscopists have more experience with improved lesion recognition, and more attention to resection of all visible lesions before RFA. As a result the requirement for rescue EMR during RFA has reduced. Although rate of progression to $\mathrm{OAC}$ is lower in the later part of the registry experience, this is not statistically significant and implies that despite advances in endoscopic imaging and technique the rate of progression remains in the region of $2-4 \%$ in these high risk patients. All collaborators of the UK RFA registry are acknowledged for their contributions to this work.

Disclosure of Interest None Declared.

\section{PTU-173 LONG TERM FOLLOW UP AFTER SUCCESSFUL RADIOFREQUENCY ABLATION FOR BARRETT'S RELATED NEOPLASIA IS ESSENTIAL TO DIAGNOSE RECURRENT DISEASE: DATA FROM THE UNITED KINGDOM PATIENT REGISTRY}

${ }^{1,2} \mathrm{RJ}$ Haidry ${ }^{*},{ }^{1} \mathrm{M}$ Banks, ${ }^{1} \mathrm{~A}$ Gupta, ${ }^{2} \mathrm{M}$ Butt, ${ }^{3} \mathrm{G}$ Fullarton, ${ }^{4} \mathrm{H}$ Smart, ${ }^{3} \mathrm{~J}$ Morris, ${ }^{5} \mathrm{R}$ Willert, ${ }^{6} \mathrm{R}$ Narayanasamy, ${ }^{1} \mathrm{M}$ Rodriguez-Justo, ${ }^{1} \mathrm{M}$ Novelli, ${ }^{1,2} \mathrm{~L}$ Lovat. ${ }^{1} \mathrm{U} C \mathrm{LH}$, London, UK; ${ }^{2} N M L C$, UCL, London, UK; ${ }^{3} G R I$, Glasgow, UK; ${ }^{4} R L U H$, Liverpool; ${ }^{5} M R I$, Manchester, UK; ${ }^{6}$ St James Hospital, Dublin, Ireland

\subsection{6/gutjnl-2014-307263.247}

Introduction Radiofrequency ablation (RFA) for patients with Barrett's oesphagus (BE) related mucosal neoplasia has been shown to be safe and effective. Endoscopic mucosal resection (EMR) for visible lesions followed by RFA is now recommended practice for these patients. Although success rates are high for disease reversal at 12 months it is appreciated that recurrence after eradication of dysplasia and intestinal metaplasia (IM) can occur in up to $25 \%$ of patients. There is still debate as to what are the optimum endoscopic follow up intervals after successful treatment.

Methods We examine prospective data from United Kingdom (UK) registry of patients undergoing RFA/EMR for BE related neoplasia over the past 5 years. We aim to establish the frequency and time of recurrences after successful treatment. Before RFA, visible lesions were removed by EMR. Thereafter patients underwent RFA every 3 months. Biopsies were taken at 12 months for clearance of dysplasia (CR-D) and BE (CR-IM). Durability and recurrence for those with successful eradication was analysed. After successful treatment patients were followed up at 3 monthly for the first year, 6 month intervals for second year and annually thereafter. Biopsies were taken from $1 \mathrm{~cm}$ below the neo $\mathrm{z}$-line and from the previously treated $\mathrm{BE}$ segment.

Results A total 508 patients have been treated. At 12 months CR-D was 85\% (428/508) and CR-IM 70\% (354/508). For those with successful outcomes at 12 months who remain in follow up, median time to their most recent biopsy is 20 months from start of treatment (range 2-72). Kaplan Meier survival statistics predict that at 5 years $75 \%$ of patients are likely to be free of dysplasia and $74 \%$ free of IM. Median time to recurrence for dysplasia is 380 days (IQR 177-619), and IM 573 days (IQR 237-816). There were 21 patients with recurrent dysplasia, $48 \%$ occurred within the first year after successful treatment, $29 \%$ in 
the second year, $14 \%$ in the third year and only $9 \%$ after 4 years.

Conclusion The majority of recurrences after successful RFA occur within the first 2 years (16/21-76\%). These date support the practice of vigilant long term follow of patients who are fit for endoscopy after treatment with RFA. More intensive and frequent follow up should take place in the first 2 years when the majority of recurrences occur. Thereafter annual follow up appears adequate. All collaborators of the UK RFA registry are acknowledged for their contributions to this work.

Disclosure of Interest None Declared.

\section{PTU-174 COST SAVING IMPLICATIONS OF NEW SURVEILLANCE GUIDELINES FOR BARRETT'S OESOPHAGUS}

R Hewett*, D Chan, A Poullis, J-Y Kang. Gastroenterology, St George's Healthcare NHS Trust, London, UK

\subsection{6/gutjnl-2014-307263.248}

Introduction The BSG have recently risk stratified Barrett's Oesophagus (BO) according to length of the $\mathrm{BO}$ segment and the presence of intestinal metaplasia (IM). Previously the recommendation was for a surveillance gastroscopy every two years. The surveillance interval recommended by the new guidelines ${ }^{1}$ now reflects the risk of developing adenocarcinoma. We aimed to quantify the potential cost saving of the implementation of the new BO surveillance guidelines.

Methods Patients with an endoscopic diagnosis BO were identified from endoscopy database records at our unit between 2009 and 2012. BO segment length was available and the presence of IM in the biopsy samples was retrievable from histology records. We allocated our patients into three groups: The 1st was those with a $\mathrm{BO}$ segment $<3 \mathrm{~cm}$ and no IM (not needing further surveillance), the 2 nd was those with a $\mathrm{BO}$ segment $<3 \mathrm{~cm}$ with IM (now needing surveillance every 5 years) and the 3 rd were those with a $\mathrm{BO}$ segment of $3 \mathrm{~cm}$ or greater (needing surveillance every 3 years). The cost of a surveillance gastroscopy is estimated to be $£ 520^{2}$ and our histopathology department advised that the cost of four quadrant biopsies was $£ 65$ (surveillance cost therefore being greater for those with longer BO segments). We first calculated the projected cost of surveillance over the next 10 years under the old guidelines. From this we subtracted the projected cost of surveillance for this period under the new guidelines.

Results 463 patients were identified who had an endoscopic diagnosis of BO. Sixty patients were excluded due to lack of data on BO length/IM.

The ten year projected cost saving for our trust by implementing the new BO surveillance guidelines was $£ 754,260$ $(£ 75,426$ per annum). There are over 150 hospital trusts in the UK that have endoscopy units, therefore even a conservative estimate is that the new $\mathrm{BO}$ guidelines will save the NHS in excess of $£ 100$ million in the next 10 years.
Conclusion New guidelines on BO surveillance will mean fewer surveillance gastroscopies need to be performed in the future. As well as giving the patients a better experience, these guidelines will result in a significant cost saving to our hospital and the NHS in general.

\section{REFERENCES}

1 Fitzgerald R, di Pietro M, Ragunath K, et al. British society of gastroenterology guidelines on the management of Barrett's oesophagus. Gut published online October 28:2013

2 Broe $M$, Barry $M$, Patchett $S$, Hill AD. Evaluating the clinical efficacy and cost effectiveness of direct access endoscopy. Surgeon 2013 Dec;11(6):304-8

Disclosure of Interest None Declared.

\section{PTU-175 DIFFERENCES IN INTESTINAL METAPLASIA IN BARRETT'S OESOPHAGUS PATIENTS FROM AN ETHNICALLY DIVERSE SOUTH LONDON POPULATION}

R Hewett ${ }^{*}$, V Chhaya, D Chan, A Poullis, J-Y Kang. Gastroenterology, St George's Healthcare NHS Trust, London, UK

\subsection{6/gutjnl-2014-307263.249}

Introduction Barrett's oesophagus (BO) is where any portion of the normal distal squamous epithelial lining has been replaced by metaplastic columnar epithelium and is a risk factor for oesophageal adenocarcinoma. The recent BSG guidelines for the endoscopic surveillance of $\mathrm{BO}$ have stratified the risk according to the length of the $\mathrm{BO}$ segment and the presence or absence of intestinal metaplasia (IM). We aimed to identify risk factors and ethnic differences for the presence of IM.

Methods We performed a retrospective database analysis in our unit which serves a large ethnically diverse southwest London population. Gastroscopy records between 2009 and 2012 were retrieved and patients with an endoscopic diagnosis of $\mathrm{BO}$ were identified. Multiple procedure reports for individual patients were removed from the analysis. Demographic information included age, sex and length of the BO segment. Patients from the Indian sub-continent were also identified, as previously described $^{1}$ The presence of IM was retrieved from the hospital pathology database and was the primary outcome measured. We performed a multivariate logistic regression analysis to determine the odds of having IM by ethnic origin and other demographics. Results 463 patients with an endoscopic diagnosis of Barrett's oesophagus were identified. Median age of diagnosis was 67.2 years (IQR: 56.7-76.6 years). Men were more likely to have an endoscopic diagnosis of $\mathrm{BO}$ than females $(71.3 \%$ vs. $29.7 \%, \mathrm{p}=$ 0.01). $9.7 \%$ of the cohort were from the Indian sub-continent.

There was an increased odds of IM amongst men although this was not statistically significant (OR 1.44 , 95\% CI: $0.94-$ $2.21, \mathrm{p}=0.09$ ). Lesion length greater than $3 \mathrm{~cm}$ compared with less than $3 \mathrm{~cm}$ was associated with a greater odds of IM (2.37, 95\% CI: $1.61-3.51, \mathrm{p}=<0.001)$. Patients from the Indian subcontinent were $70 \%$ less likely to have IM compared to other ethnicities (OR 0.32, 95\% CI: 0.16-0.61, p = 0.001).

\begin{tabular}{|c|c|c|c|c|c|}
\hline & Patients & Old cost of surveillance (10 y) & New cost of surveillance $(10 \mathrm{y})$ & Cost saving over $10 y$ & Mean cost saving per annum \\
\hline$<3 \mathrm{~cm}$, no IM & 97 & $£ 283,735$ & f0 & f283,735 & $£ 28,373$ \\
\hline$<3 \mathrm{~cm}$, with IM & 103 & f301,275 & $£ 120,510$ & f180,765 & $£ 18,076$ \\
\hline$>3 \mathrm{~cm}$ & 203 & f725,425 & $f 434,655$ & $\mathrm{f} 290,770$ & $£ 29,077$ \\
\hline All patients & 403 & $£ 1,309,425$ & f555,165 & $f 754,260$ & $f 75,426$ \\
\hline
\end{tabular}

